\title{
Study on batching and picking optimization of marine outfitting pallets
}

\author{
$D G \mathrm{Zhao}^{1}, Y \mathrm{Jiang}^{2, *}, J W \mathrm{Bao}^{1}, J Q \mathrm{Wang}^{2}$, and $H \mathrm{Jia}^{2}$ \\ ${ }^{1}$ Shanghai Waigaoqiao Shipbuilding Co., Ltd., Shanghai 200137, China \\ ${ }^{2}$ College of Shipbuilding Engineering, Harbin Engineering University, Harbin 150001, China
}

\begin{abstract}
Outfitting pallet picking involves the retrieval of items from their storage sites in shipbuilding enterprises. A major issue in manual pallet picking operations is the transformation of outfitting pallets into picking batches (pallet batching). Considering the influence to the subsequent distribution and production processes, a mathematical model for the batching and picking problem of outfitting pallets is formulated with the objective of minimizing the total tardiness of all pallets. According to the characteristics of outfitting pallet picking operations, an Improved Genetic Algorithm (IGA) is proposed. A reversal operator is specially introduced to increase the local search ability of the standard genetic algorithm and speed up the evolution. Benchmarked against the solutions produced by the Earliest Due Date (EDD) rule, the performance of IGA is studied under different picking operations with different workloads and tightness of due dates. A series of numerical experiments are carried out to verify the researches. The results clearly show that IGA is competitive since it improves the solutions by $68.5 \%$, on average, relative to the EDD.
\end{abstract}

\section{Introduction}

Marine outfitting refers to the installation of equipments on board other than the hull and power plant. The quantities of outfitting usually accounts for $50 \%$ to $60 \%$ of the total shipbuilding project volume [1]. The refined production management under the modern shipbuilding mode puts forward higher requirements for the picking and distribution of outfitting pallets. Delays in pallet picking operations will not only affect the distribution of pallets, but also disrupt the implementation of outfitting plans.

Outfitting pallet batching and picking problem has not been discussed frequently in the literature, but several solution approaches to the relevant problems have been proposed so far. Henn \& Schmid [2] applied Iterated Local Search and Attribute-Based Hill Climber metaheuristics to solve the Order Batching and Sequencing Problem (OBSP). Compared to the solutions constructed with the Earliest Due Date (EDD) rule, the solutions obtained using these two methods increased by $46 \%$ on average. Menéndez et al. [3] have studied the OBSP using the General Variable Neighborhood Search variant, which introduced three different neighbourhood structures to improve the solution results.

*Corresponding author: jeangyan@hrbeu.edu.cn 
Additionally, several types of genetic algorithms (GA) have been proposed for the related problems. Hsu et al. [4] suggested a GA-based order batching method, the objective of which was to minimize the distance of the picking path. Tsai et al. [5] have proposed a batch picking model that simultaneously considers the travel cost and earliness and tardiness penalty. They then solved it using GA_BATCH and GA_TSP algorithms. The former searches for the optimal batching results with the minimal sum of the travel cost and earliness and tardiness penalties, while the latter finds the shortest picking path.

This paper is organized as follows: Section 2 gives a brief introduction to marine outfitting pallet batching and picking problem. Moreover, a mathematical model for the problem is presented. Section 3 describes the implementation of the proposed Improved Genetic Algorithm (IGA) in detail. In Section 4, a series of numerical experiments are designed and carried out to test the performance of IGA. Finally, the relevant conclusion is presented in Section 5.

\section{Marine outfitting pallet batching and picking problem}

\subsection{Problem description}

Marine outfitting supplies are characterized by large quantity and variety. According to the storage conditions of shipbuilding enterprises at present, this paper studies a manual picking area with parallel picking aisles and two cross aisles, as shown in figure1. The depot is situated in front of the leftmost picking aisle, which is the departure station and destination of a picking path. Outfitting supplies are stored in accordance with the frequency of demand and divided into three classes. Class A covers $50 \%$ of the demanded items that are uniformly stored in picking aisle 1, class B includes $30 \%$ of items stored in picking aisles 2,3 , and class $\mathrm{C}$ contains $20 \%$ of items stored in other three picking aisles.

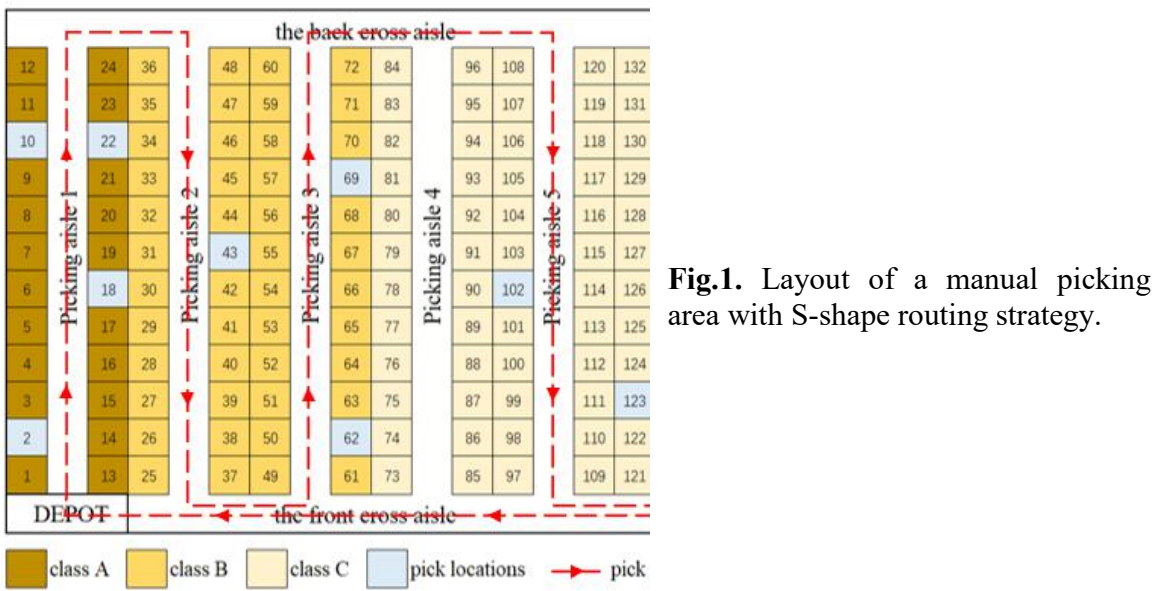

The picking area has only one picker that all batches must begin successively. The picker adopts a S-shape routing strategy. He/She enters and traverses an entire picking aisle if it contains requested items, then proceeds to the next picking aisle needed. One exception is that if the number of served picking aisles is odd, the picker will traverse the last picking aisle to the farthest picking site and return to the depot [6]. The capacity of the picking device is measured by the maximum number of items that can be picked using the device in one tour. All outfitting pallets cannot be split. 


\subsection{Model formulation}

For the above pallet picking operations, the following parameters and variables are introduced:

$S \quad$ set of outfitting pallets $(S=\{1,2, \ldots, n\})$

$B$ set of all feasible picking batches

$K$ set of positions where a batch can be scheduled $(K=\{1,2, \ldots, m\})$

$C$ capacity of the picking device

$c_{i} \quad$ number of requested items in pallet $i \in S$

$d_{i} \quad$ due date of pallet $i \in S$

$M$ a extremely large positive number

$c t_{k}$ completion time of the batch scheduled at position $k \in K$

$p t_{j}$ processing time of batch $j \in B$

$t d_{i k}$ tardiness of pallet $i \in S$ if included in the batch scheduled at position $k \in K$

$a_{i j}$ variable for indicating whether pallet $i \in S$ is included in batch $j \in B\left(a_{i j}=1\right)$ or $\operatorname{not}\left(a_{i j}=0\right)$

$x_{i k}$ variable for indicating whether pallet $i \in S$ is allocated to the batch scheduled at position $k \in K\left(x_{i k}=1\right)$ or not $\left(x_{i k}=0\right)$

$y_{i k}$ variable for indicating whether batch $j \in B$ scheduled at position $k \in K\left(y_{i k}=1\right)$ or $\operatorname{not}\left(y_{i k}=0\right)$

The problem can then be formulated as follows:

$$
\begin{array}{ll}
\min \quad & \sum_{i \in S} \sum_{k \in K} t d_{i k} \\
& \sum_{j \in B} y_{j k} \leq 1, \forall k \in K \\
& \sum_{k \in K} y_{j k}=1, \forall j \in B \\
& \sum_{j \in B} a_{i j} y_{j k}=x_{i k}, \forall i \in S, k \in K \\
& \sum_{k \in K} x_{i k}=1, \forall i \in S \\
& \sum_{i \in S} c_{i} a_{i j} \leq C, \forall j \in B \\
& \sum_{j \in B} p t_{j} y_{j 1} \leq c t_{1} \\
& c t_{k-1}+\sum_{j \in B} p t_{j} y_{j k} \leq c t_{k}, \forall k \in K \backslash\{1\} \\
& c t_{k}-t d_{i k} \leq d_{i}+M\left(1-x_{i k}\right), \forall i \in S, k \in K \\
& t d_{i k} \geq 0, \forall i \in S, k \in K \\
& a_{i j}, x_{i k}, y_{j k} \in\{0,1\}, \forall \mathrm{i} \in \mathrm{S}, \mathrm{j} \in \mathrm{B}, \mathrm{k} \in \mathrm{K}
\end{array}
$$

The objective function (1) minimizes the total tardiness of all pallets. Constraint (2) assures that at most one batch is assigned to a particular position, while (3) ensures that each batch is picked. The consistency among pallets and batches scheduled at any position is guaranteed by satisfying (4). Restriction (5) ensures that each pallet is allocated to one batch. Constraint (6) indicates the capacity of the picking device can not be exceeded. Inequalities (7) and (8) determine the completion time of the batch. Conditions (9) and (10) restrict the tardiness of each pallet. 


\section{Improved genetic algorithm}

\subsection{General principle}

Genetic algorithms have been widely and successfully implemented due to their implicit parallelism and global solution space search [7]. To effectively resolve the problem modelled in section 2, an Improved Genetic Algorithm is proposed, as demonstrated in figure 2 .

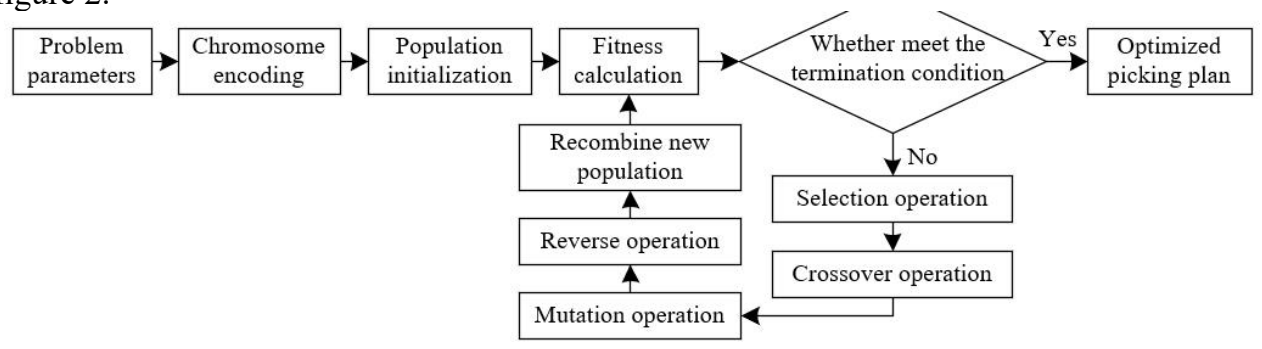

Fig. 2. The solution process of the Improved Genetic Algorithm.

\subsection{Adaption for the problem}

\subsubsection{Encoding and decoding}

A pallet-based encoding method is suggested to avoid generating infeasible solutions. The integer encoded in the chromosome symbolizes the sequence number of the pallet. For a

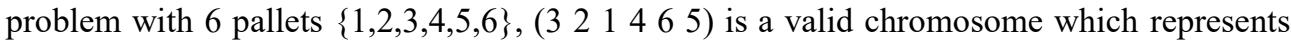
that the picking priority of pallets is $3>2>1>4>6>5$. The decoder is set to batch pallets according to the picking priority and by the capacity of the picking device.

\subsubsection{Fitness function}

Each feasible solution corresponds to a matching fitness, which determines the quality of the reaction to other solutions of the population. According to the constraints of the problem, the fitness function we want to maximize can be defined as

$$
\text { fit }_{i}=1 / \text { tar }_{i}
$$

where $f i t_{i}$ and $\operatorname{tar}_{i}$ are the fitness value and tardiness value of a feasible solution $i$.

\subsubsection{Selection}

In selection operation, a set number of solutions are selected from the current generation to be copied into the next generation. A stochastic universal sampling method is adopted [8]. The probability of solution i to be selected is

$$
p_{i}=f_{i} t_{i} / \sum f i t_{i}
$$

\subsubsection{Crossover and mutation}

A partially-mapped crossover method is utilized in crossover operation. While using this approach, a portion of one parent chromosome is mapped onto a portion of another parent chromosome, and the rest of the information is exchanged [9]. The mutation operation lets 
each component in the chromosome has a probability (i.e. the mutation rate) to exchange its information with another component selected.

\subsubsection{Inverse operator}

To enhance the local search ability of the algorithm, an inverse operator is introduced, which can reverse the sequence of the information between two selected components in a solution. Additionally, the inversed solution can only be adopted when it is superior to the original.

\subsubsection{Termination condition}

In each generation, the best objective value is recorded. The termination condition of IGA is defined as the minimum maintenance generations for the best objective value.

\section{Experiments}

To verify the performance of the suggested algorithm, a series of numerical experiments have been carried out. In these experiments, the picking area mentioned in section 2 is considered. The length of each storage site is assumed to be one length unit (LU). The picker must move one LU vertically when leaving a picking aisle from the first or the last storage site and move 5 LUs horizontally to the next picking aisle. The processing time of a picking batch is composed of the travel time, search time, pick time and setup time [10]. For travel time, we assume that it takes $2 \mathrm{~s}$ for a vehicle to drive $1 \mathrm{LU}$. Moreover, it needs $10 \mathrm{~s}$ to find and pick an item from the corresponding storage site. Each tour requires 1 minutes spent on administration and setup.

In the following experiments, the set of instances considers 5 different number of outfitting pallets $(n=\{20,40,60,80,100\})$, where the number of items per pallet is evenly distributed in $\{2,3,4,5\}$. The capacity of the picking device is set to 10 items. The distribution of the due date for all pallets is a truncated normal distribution, which falls within the interval as follows,

$$
\left[\min _{i \in S} p t_{i}^{S}, 2(1-\text { MTCR }) \sum_{i \in S} p t_{i}^{S}+\min _{i \in S} p t_{i}^{S}\right], 0 \leq \text { MTCR } \leq 1
$$

where $p t_{i}^{S}$ denotes the processing time of the pallet $i \in S$ if processed separately [11]. The values of the modified traffic congestion rate (MTCR) considered are: $0.60,0.65,0.70$ and 0.75 . We obtain a total of 20 problem classes, of which each has generated 50 instances.

In order to provide a method against which IGA can be benchmarked, the Earliest Due Date rule is introduced. Under this rule, all outfitting pallets are sorted and picked in descending order according to their due date. figure 3 shows the development of optimal value in the iterative process of IGA.

Table 1 describes the experimental results for the total tardiness (tar), total processing time, i.e., makespan (mak) and facility utilization (uti). Moreover, the improvement of the solution quality (imp) obtained by the evaluated algorithms is presented. All entries in table 1 are the average solution values of the 50 implemented instances in each problem class. 


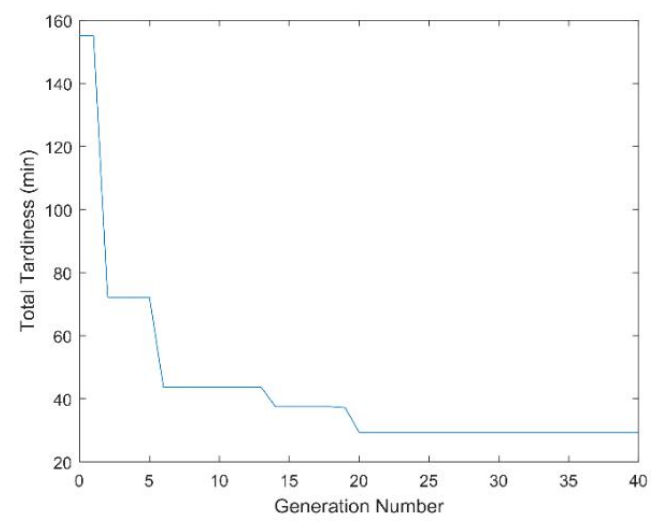

Fig.3. Example for the optimization process of using IGA to solve the instance(when $n=40$, $\mathrm{MTCR}=0.65)$.

Table 1. The results of numerical experiments.

\begin{tabular}{|c|c|c|c|c|c|c|c|c|}
\hline \multirow[b]{2}{*}{$n$} & \multirow[b]{2}{*}{ MTCR } & \multicolumn{3}{|c|}{ Earliest Due Date rule } & \multicolumn{4}{|c|}{ Improved Genetic Algorithm } \\
\hline & & $\operatorname{tar}(\min )$ & $\operatorname{mak}(\min )$ & uti(\%) & $\operatorname{tar}(\min )$ & $\operatorname{mak}(\min )$ & uti(\%) & $\operatorname{imp}(\%)$ \\
\hline \multirow{4}{*}{20} & 0.60 & 15.1 & 56.9 & 84.0 & 4.9 & 54.0 & 85.3 & 67.2 \\
\hline & 0.65 & 43.9 & 56.7 & 84.3 & 17.1 & 52.3 & 87.0 & 61.0 \\
\hline & 0.70 & 102.7 & 58.5 & 82.6 & 48.2 & 51.5 & 89.5 & 53.0 \\
\hline & 0.75 & 175.4 & 57.7 & 83.7 & 90.8 & 49.8 & 90.0 & 48.2 \\
\hline \multirow{4}{*}{40} & 0.60 & 36.7 & 115.2 & 84.6 & 5.0 & 109.2 & 86.9 & 86.3 \\
\hline & 0.65 & 130.1 & 113.1 & 85.0 & 26.2 & 102.4 & 88.7 & 79.7 \\
\hline & 0.70 & 379.7 & 113.5 & 84.6 & 141.1 & 99.1 & 90.3 & 62.8 \\
\hline & 0.75 & 615.4 & 112.7 & 85.1 & 293.2 & 97.1 & 91.4 & 52.3 \\
\hline \multirow{4}{*}{60} & 0.60 & 31.2 & 169.5 & 85.8 & 4.1 & 165.0 & 86.8 & 86.8 \\
\hline & 0.65 & 257.0 & 171.2 & 85.6 & 48.4 & 156.0 & 89.3 & 81.1 \\
\hline & 0.70 & 718.2 & 168.4 & 85.8 & 274.5 & 147.8 & 91.0 & 61.7 \\
\hline & 0.75 & 1370.5 & 167.8 & 85.8 & 685.4 & 145.7 & 91.1 & 49.9 \\
\hline \multirow{4}{*}{80} & 0.60 & 29.6 & 223.3 & 86.3 & 1.0 & 219.7 & 86.8 & 96.5 \\
\hline & 0.65 & 400.5 & 228.1 & 85.9 & 67.0 & 208.0 & 90.0 & 83.2 \\
\hline & 0.70 & 1183.7 & 223.9 & 85.9 & 424.7 & 197.2 & 91.3 & 64.1 \\
\hline & 0.75 & 2515.5 & 228.0 & 86.2 & 1287.8 & 198.2 & 91.5 & 48.8 \\
\hline \multirow{4}{*}{100} & 0.60 & 39.6 & 281.2 & 85.4 & 1.3 & 277.5 & 86.0 & 96.5 \\
\hline & 0.65 & 524.8 & 282.1 & 86.3 & 89.17 & 260.0 & 89.5 & 83.0 \\
\hline & 0.70 & 2031.3 & 282.2 & 86.1 & 836.7 & 249.5 & 91.0 & 58.8 \\
\hline & 0.75 & 3814.7 & 281.3 & 86.2 & 1894.5 & 244.8 & 91.4 & 50.3 \\
\hline \multicolumn{2}{|c|}{ Average } & & & & & & & 68.5 \\
\hline
\end{tabular}

It is obvious that the total tardiness increases with an increasing number of outfitting pallets. Moreover, when the number of pallets is the same, the total tardiness is increased as MTCR increases. The total tardiness gained by IGA improves by $68.5 \%$ over that of EDD on average, which is a significant improvement in actual application. In addition, the utilization rate of the picking device in the IGA is generally greater than that in the EDD. When MTCR is small, IGA is able to postpone a pallet with an early due date to a later time point if the total tardiness can be reduced. However, with the increase of MTCR, most pallets cannot meet their due dates. In this case, the total tardiness can only be reduced by increasing the facility utilization and reducing the makespan. 


\section{Conclusion}

In this work, we tackled the batching and picking problem of outfitting pallets, which is an important part in outfitting process. An Improved Genetic Algorithm that introduced a reversal operator to enhance the local search capability and accelerate the evolution was proposed to the problem. One thousand instances under twenty different problem classes were implemented in numerical experiments. The results show that IGA can effectively reduce the total tardiness in pallet picking operations, and the solutions generated by IGA significantly outperform those of the EDD. It is worth noting that when the number of outfitting pallets $(n)$ is small, an optimal picking plan can generally be obtained by IGA. When $n$ is relatively large, IGA can only get an approximate solution. In this case, the optimization result can be improved by increasing the size of chromosome population and raising the termination condition. In actual outfitting pallet picking operations, the problems encountered may be different from the problem model formulated in this paper. In these circumstances, the problem model should be modified according to the practical conditions, but the core idea and processing method of the IGA can continue to be utilized.

\section{Acknowledgement}

The authors would like to thank the editor and anonymous reviewers for their comments and suggestions. This research is partially supported by programs "Large Cruise Research and Development Project (2017)" and "Research on Key Common Technologies towards Smart Manufacturing in Shipbuilding Industry" funded by Ministry of Industry and Information Technology of the People's Republic of China (No. 2016543), and this paper is also supported by National Natural Science Foundation of China (No. 51679059).

\section{References}

1. Zhou Y 2011 Research on the application of ship outfitting technology and pallet management in shipbuilding (Harbin: Harbin Engineering University)

2. Henn S and Schmid V 2013 Metaheuristics for pallet batching and sequencing in manual pallet picking systems Comput. Ind. Eng. 66 338-51

3. Menéndez B, Bustillo M, Pardo E G and Duarte A 2017 General variable neighborhood search for the pallet batching and sequencing problem Eur. J. Oper. Res. 263 82-93

4. Hsu C M, Chen K Y and Chen M C 2005 Batching pallets in warehouses by minimizing travel distance with genetic algorithms Comput. Ind. 56 169-78

5. Tsai C Y, Liou J and Huang T M 2008 Using a multiple-ga method to solve the batch picking problem: considering travel distance and pallet due time Int. J. Prod. Res. 46 6533-55

6. Hall R 1993 Distance approximations for routing manual pickers in a warehouse IIE. Trans. 25 76-87

7. He Y, Xu S, Zhu K, Liu T and Li Y 2008 A genetic-neural method of optimizing cutoff grade and grade of crude ore Expert. Syst. Appl. 36 7617-23

8. Baker J E 1985 Adaptive selection methods for genetic algorithms. Proc. First International Conference on Genetic Algorithms and Their Applications pp 867-72

9. Ting C K, Su C H and Lee C N 2010 Multi-parent extension of partially mapped crossover for combinatorial optimization problems Pergamon Press, Inc. 
10. Scholz A, Schubert D and Wäscher G 2017 Order picking with multiple pickers and due dates -simultaneous solution of order batching, batch assignment and sequencing, and picker routing problems Eur. J. Oper. Res. 263 461-78

11. Elsayed E A, Lee M K, Kim S and Scherer E 1993 Sequencing and batching procedures for minimizing earliness and tardiness penalty of order retrievals Int. J. Prod. Res. 31 727-38 